\title{
The influence of peripheral vision induced by moving people on postural control in healthy adults
}

\author{
Soo-Han Kim, Du-Jin Park* \\ Department of Physical Therapy, Kaya University, Gimhae, Korea
}

This study was conducted to examine the influence of the peripheral vision (PV) induced by moving people on postural control in healthy adults. The subjects consisted of 12 healthy adult volunteers $(5$ males, 7 females) who had been informed of the study purpose and procedure. The visual interventions were composed of three types. PV1 and PV2 were stimulated using a one-way vertical striped pattern and a twoway vertical striped pattern, respectively. To embody the features of moving people reflected in the mirrors, researchers recorded movements of people or objects provided by mirrors on video image. In this study, this was named PV3. The subjects were exposed to each of the visual stimuli for $3 \mathrm{~min}$ in a random order, and their postural control was then evaluated. All the subjects were allowed to practice once prior to performing the one leg stand test, functional reaching test and body sway test. All the evaluations were made before and after the visual intervention, and the subjects rested for 30 min between each intervention. PV3 ranked second in before and after differences of trace length and velocity and had no significant difference from PV2, demonstrating that the PV3, as well as PV2, affected the amount and velocity of body sway. In addition, the standard deviation velocity, trace length and velocity values of PV3 were higher than the PV1 values. Therefore, the treatment of those who have difficulty with postural control and balance maintenance should take place in a controlled therapeutic environment.

Keywords: Peripheral vision, Postural control, Moving people

\section{INTRODUCTION}

Postural stability depends on postural control, which refers to the ability to maintain the centre of gravity of the body within a certain space domain. Postural control is essential to lead an independent life, perform activities of daily living, and ensure a correct gait (Tinetti et al., 1988). Anticipatory postural control is required while performing voluntary movements and motions to minimise centre of gravity movements (Nouillot et al., 1992; Wade and Jones, 1997). For precise postural control, information provided by the visual, vestibular and somatosensory system is very important (Massion, 1992).

Among the three sensory systems, the visual system primarily responds to high-frequency movement (Wade and Jones, 1997). However, it is also sensitive to low-frequency stimulation by postural sway $(0.5 \mathrm{~Hz}$ or smaller) and gait activity $(1.0 \mathrm{~Hz}$ or smaller) (Wade and Jones, 1997). The interception of visual system may trigger postural sway of 50\% or higher (Edwards, 1946), and decreased vision increases postural sway and falls (Paulus et al., 1984; West et al., 2002). In addition, a moving visual environment can lead to visual confusion, triggering postural change, motion sickness and postural imbalance (Redfern and Furman, 1994). Thus, the visual senses play an important role in the human body's balance and postural control.

In humans, the visual field is divided into central vision (CV) and peripheral vision (PV). CV detects objects in the gaze direction, and PV detects the existence of circumjacent objects, although imperfectly (Straube et al., 1994). PV is closely related to Y-cells, which are evenly distributed over a large area in the retina and sensitive to the movement of objects (Dix et al., 2004). PV can give rise to vection, the perception of self-motion, which is a kind of optical illusion that involves the feeling of movement in a static condition due to dynamic visual stimuli. PV plays a greater role in vection than $\mathrm{CV}$; it is also more important than $\mathrm{CV}$ in
*Corresponding author: Du-Jin Park (iD http://orcid.org/0000-0002-5658-3343 Department of Physical Therapy, Kaya University, 208, Samgye-ro, Gimhae 50830, Korea

Tel: +82-55-330-1047, Fax: +82 55-330-1146, E-mail: djpark35@kaya.ac.kr Received: August 17, 2016 / Accepted: September 5, 2016
This is an Open Access article distributed under the terms of the Creative Commons Attribution Non-Commercial License (http://creativecommons.org/licenses/by-nc/4.0/) which permits unrestricted non-commercial use, distribution, and reproduction in any medium, provided the original work is properly cited. 
sensing the movement of objects and postural sway (Andersen and Braunstein, 1985; Delorme and Martin, 1986; Dix et al., 2004).

A previous PV visual stimulation study reported that a vertical striped pattern greatly affected postural changes (Kim et al., 1996). More recent research reported that a two-way vertical striped pattern had a greater impact on postural changes and PV and $\mathrm{CV}$ than a one-way vertical striped pattern, with the greatest postural sway detected with the two-way vertical striped pattern (Park, 2016). As stimulation of the PV is closely related to postural control and sway, those who have problems with PV have problems with postural control.

The walls of many rehabilitation treatment rooms in Korea are covered in mirrors. However, this type of environment may not be suitable for the treatment of individuals who have difficulty with postural control and perception, as the reflected movements of people or objects stimulate the PV, thereby increasing postural sway. Accordingly, this study was conducted to examine the influence of the PV induced by moving people on postural control in healthy adults.

\section{MATERIALS AND METHODS}

\section{Subjects}

The subjects in this study were five young males and seven young females who listened to the purpose and procedure of the experiment and voluntarily consented to participate in the study. The average ages, weights, heights and body mass indexes of the subjects were $22.08 \pm 3.87$ years, $55.50 \pm 10.06 \mathrm{~kg}, 167.33 \pm 8.22$ $\mathrm{cm}$, and $19.72 \pm 2.64 \mathrm{~kg} / \mathrm{m}^{2}$, respectively. Individuals who took drugs that could affect postural control or who had a disease that could influence vision and postural control were excluded from the experiment. Ethical approval was granted by Kaya University Institutional Review Board.

\section{Visual interventions and methods}

The visual interventions were composed of three types (Fig. 1). PV1 and PV2 were stimulated using a one-way vertical striped

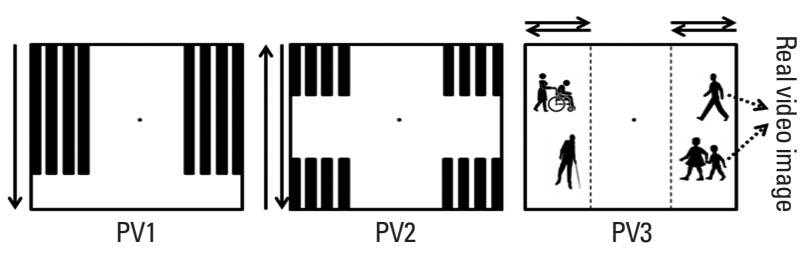

Fig. 1. The visual interventions using peripheral vision variations. PV, peripheral vision. pattern and a two-way vertical striped pattern, respectively. To embody the features of moving people reflected in the mirrors, researchers recorded movements of people or objects provided by mirrors on video image. In this study, this was named PV3. All the subjects put on equipment that controlled each of PV stimulations. They then received the visual interventions while standing $5 \mathrm{~m}$ away from the screen. They were exposed to each of the visual stimuli for $3 \mathrm{~min}$ in a random order, and their postural control was then evaluated.

In this study, the subjects performed one leg stand test (OLS). During the OLS, the subjects had to maintain their balance as best they could by supporting their body with the dominant leg, with their eyes open (OLSO) and closed (OLSC). The maximal duration of the OLS was $30 \mathrm{sec}$. The subjects' dynamic balance ability was assessed using a functional reaching test (FRT). In addition, body sway was evaluated. To analyse body sway, the BIORescue (RM Ingeierie, Rodez, France) device was used. All the subjects were allowed to practice once prior to performing the OLS, FRT and body sway test. All the evaluations were made before and after the visual interventions, and the subjects rested for 30 min between each intervention.

\section{Statistical analysis}

To examine the influences of $\mathrm{PV}$ induced moving people on postural control in healthy adults, one-way repeated analysis of variance was used. As a post hoc test, the Bonferroni correction was utilized, and the significance level was set at $\alpha=0.05$. The collected data were analysed using IBM SPSS ver. 18.0 (IBM Co., Armonk, NY, USA).

\section{RESULTS}

With the eyes open and closed, there was no significant difference among the visual interventions in OLS and FRT (Table 1). There was a significant difference among the visual interventions in the trace length (TL; Fig. 2) and velocity (Fig. 3), with the greatest increase in PV2 (Table 2). The standard deviation velocity (SDV) of PV1 and PV2 was significantly different (Fig. 4). There was also a significant difference in the SDV between PV1 and PV2 and between PV1 and PV3 (Table 2).

\section{DISCUSSION}

PV has a greater effect on body balance than CV. Many studies reported that visual stimulations involving $\mathrm{PV}$ in balance training 
Table 1. Effects of the visual interventions in the FRT, OLSO, and OLSC

\begin{tabular}{lcccc}
\hline Variable & PV1 & PV2 & PV3 & $F$ \\
\hline FRT (cm) & & & & \\
$\quad$ Before & $89.16 \pm 5.54$ & $89.16 \pm 5.54$ & $89.16 \pm 5.54$ & \\
After & $84.85 \pm 4.68$ & $84.98 \pm 4.67$ & $86.83 \pm 5.22$ & \\
Difference & $-4.31 \pm .93$ & $-4.18 \pm 3.74$ & $-2.33 \pm 2.31$ & 2.265 \\
OLSO (sec) & & & & \\
Before & $29.60 \pm 1.40$ & $29.60 \pm 1.40$ & $29.60 \pm 1.40$ & \\
After & $29.17 \pm 2.88$ & $29.45 \pm 1.42$ & $29.93 \pm 0.23$ & \\
Difference & $-0.43 \pm 1.49$ & $-0.15 \pm 0.54$ & $0.34 \pm 1.44$ & 0.806 \\
OLSC (sec) & & & & \\
Before & $21.84 \pm 6.60$ & $21.84 \pm 6.60$ & $21.84 \pm 6.60$ & \\
After & $20.24 \pm 6.65$ & $19.49 \pm 7.38$ & $21.18 \pm 6.99$ & \\
Difference & $-1.60 \pm 7.52$ & $-2.35 \pm 7.46$ & $-0.67 \pm 2.75$ & 0.441
\end{tabular}

Values are presented as mean \pm standard deviation.

FRT, functional reaching test; OLSO, one leg standing with open eyes; OLSC, one leg standing with closed eyes; PV, peripheral vision.

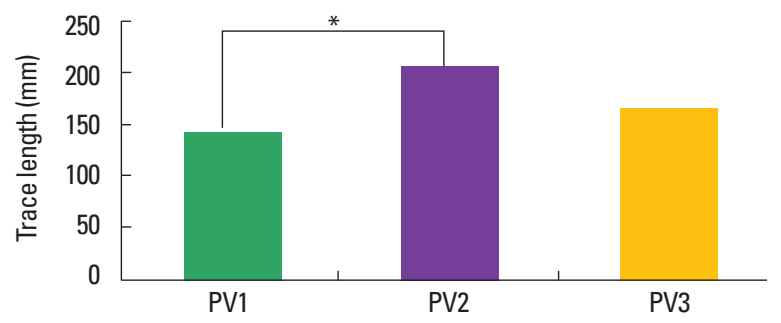

Fig. 2. Effects of the visual interventions on the trace length. PV, peripheral vision. *Significant difference between conditions.

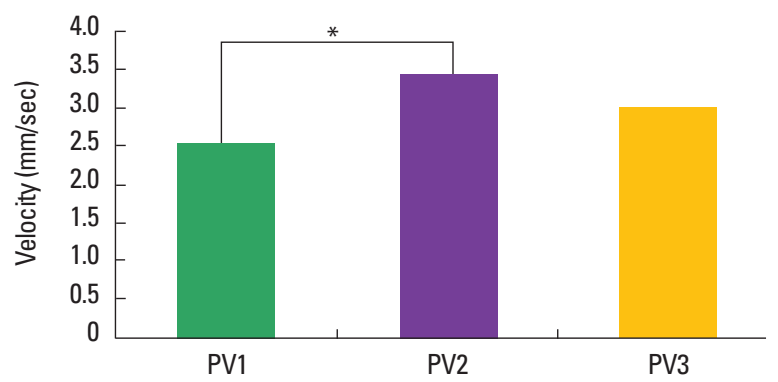

Fig. 3. Effects of the visual interventions on the velocity. PV, peripheral vision. * Significant difference between conditions.

were more useful in diverse rehabilitation areas than visual stimulations involving CV (Kim et al., 1996; Park, 2016). Recent research suggested that the movements of people close by in a space surrounded by mirrors could have adverse effects on subjects receiving treatment for postural control (Park, 2016). Accordingly, this study aimed to examine how the PV3 shown by mirrors affected postural control.

In previous research (Park, 2016), there was no significant dif-
Table 2. Effects of the visual interventions on the amount of body sway

\begin{tabular}{|c|c|c|c|c|}
\hline Variable & PV1 & PV2 & PV3 & $F$ \\
\hline \multicolumn{5}{|l|}{$\mathrm{TL}^{*}(\mathrm{~mm})$} \\
\hline Before & $509.44 \pm 98.27$ & $509.44 \pm 98.27$ & $509.44 \pm 98.27$ & \\
\hline After & $651.31 \pm 129.51$ & $713.89 \pm 119.71$ & $673.74 \pm 124.69$ & \\
\hline Difference & $141.87 \pm 60.53^{\mathrm{a})}$ & $204.44 \pm 27.71^{\mathrm{bl}}$ & $164.30 \pm 52.99^{\mathrm{a}, \mathrm{b})}$ & 10.368 \\
\hline \multicolumn{5}{|c|}{$\mathrm{SDV}^{*}(\mathrm{~mm} / \mathrm{sec})$} \\
\hline Before & $4.71 \pm 1.28$ & $4.71 \pm 1.28$ & $4.71 \pm 1.28$ & \\
\hline After & $6.38 \pm 1.67$ & $7.27 \pm 1.38$ & $7.09 \pm 1.05$ & \\
\hline Difference & $1.67 \pm 0.80^{\mathrm{a}}$ & $2.56 \pm 0.76^{b)}$ & $2.38 \pm 0.49^{b)}$ & 7.355 \\
\hline \multicolumn{5}{|c|}{ Velocity* (mm/sec) } \\
\hline Before & $8.49 \pm 1.64$ & $8.49 \pm 1.64$ & $8.49 \pm 1.64$ & \\
\hline After & $10.99 \pm 2.31$ & $11.92 \pm 2.16$ & $11.50 \pm 2.10$ & \\
\hline Difference & $2.51 \pm 1.11^{a)}$ & $3.43 \pm 0.85^{b l}$ & $3.01 \pm 1.10^{\mathrm{a}, \mathrm{bl}}$ & 5.318 \\
\hline
\end{tabular}

$\mathrm{TL}$, trace length; SDV, standard deviation velocity; PV, peripheral vision.

a,b $V$ Values within a row with different superscripts are significantly different $(P<0.05)$.

${ }^{*}$ Significant difference between conditions.

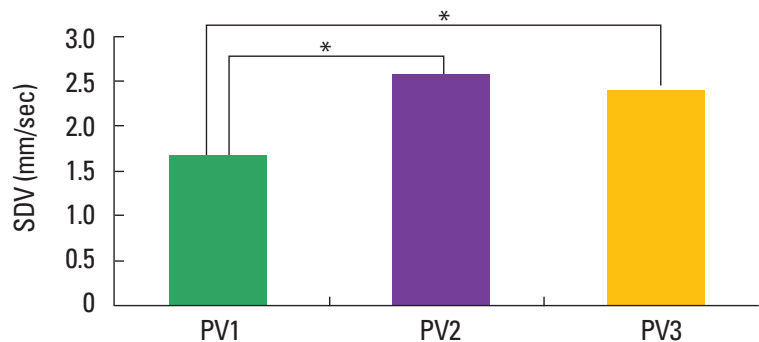

Fig. 4. Effects of the visual interventions on the standard deviation velocity (SDV). PV, peripheral vision. * Significant difference between conditions.

ference between a one-way linear pattern and two-way linear pattern using PV in FRT and OLSC. In the present study, there was no significant difference between the two PV patterns recorded during the FRT, OLSO, and OLSC. This finding supports the results of previous research, which found no significant difference between a one-way linear pattern and two-way linear pattern using PV in FRT and OLSC. In addition, there was no significant difference among the three PV patterns. As the PV3 is a visual intervention based on PV, the PV3 likely had a similar effect on the two PV patterns and the FRT, OLSO, and OLSC.

In the present study, the TL was the length of the centre of pressure (COP) line, and the velocity was the average speed of the COP. In this study, the TL and velocity values of PV2 were significantly greater than those of PV1. This means that the two-way linear pattern had a greater effect than the one-way linear pattern on the amount and speed of body sway. In addition, PV3 ranked second in before and after differences of TL and velocity and had no significant difference from PV2, demonstrating that the PV3, 
as well as PV2, affected the amount and velocity of body sway, possibly due to difficulty maintaining balance.

In the present study, SDV represented the standard deviation of the speed of body sway, with higher values indicative of greater body sway. In this study, the SDV of PV2 and PV3 was significantly higher than the SDV of PV1. Previous studies reported that a one-way linear pattern had a greater effect on PV and postural sway than on CV (Kim et al., 1996; Park, 2016). In the present study, the SDV, TL and velocity values of PV3 were higher than the PV1 values.

As illustrated above, when individuals with diverse complaints are treated together in an environment surrounded by mirrors, the environment may have adverse effects on those who have difficulty maintaining balance and postural control. Therefore, the treatment of those who have difficulty with postural control and balance maintenance should take place in a controlled therapeutic environment. Mirrors may help subjects to perceive and correct their posture, thereby aiding postural control. In training involving the use of mirrors, such training should be provided in a therapy room where PV3 is controlled. CV also plays an important role in perceiving self-motion (Andersen and Braunstein, 1985; Paulus et al., 1984). If the size of the mirrors is adjusted to the range of $\mathrm{CV}, \mathrm{PV} 3$ may be minimal, and it will be effective in balance training.

This study has some limitations. As it consisted of young healthy males and females, the findings may not be generalizable. In addition, to apply the same PV3 intervention, the images of the PV3 reflected in the mirrors were photographed. Therefore, it is different from PV3 illuminated directly through mirrors. Future studies are needed of the effects of actual PV3 provided through mirrors on the balance of subjects who have difficulty with postural control.

\section{CONFLICT OF INTEREST}

No potential conflict of interest relevant to this article was reported.

\section{ACKNOWLEDGMENTS}

This work was supported by Kaya University Research Fund.

\section{REFERENCES}

Andersen GJ, Braunstein ML. Induced self-motion in central vision. J Exp Psychol Hum Percept Perform 1985;11:122-132.

Delorme A, Martin C. Roles of retinal periphery and depth periphery in linear vection and visual control of standing in humans. Can J Psychol 1986;40:176-187.

Dix A, Finlay J, Abowd GD, Beale R, editors. Human-computer interaction. 3rd ed. Harlow: Pearson Prentice Hall; 2004.

Edwards AS. Body sway and vision. J Exp Psychol 1946;36:526-535.

Kim HS, Kim DW, Cha EJ, Kim YH, Kim NG. Human postural balance control by visual stimulation. J KOSOMBE 1996;17:417-426.

Massion J. Movement, posture and equilibrium: interaction and coordination. Prog Neurobiol 1992;38:35-56.

Nouillot P, Bouisset S, Do MC. Do fast voluntary movements necessitate anticipatory postural adjustments even if equilibrium is unstable? Neurosci Lett 1992;147:1-4.

Park DJ. Effect of visual stimulus using central and peripheral visual field on postural control of normal subjects. J Phys Ther Sci 2016;28:17691771.

Paulus WM, Straube A, Brandt T. Visual stabilization of posture. Physiological stimulus characteristics and clinical aspects. Brain 1984;107(Pt 4):1143-1163.

Redfern MS, Furman JM. Postural sway of patients with vestibular disorders during optic flow. J Vestib Res 1994;4:221-230.

Straube A, Krafczyk S, Paulus W, Brandt T. Dependence of visual stabilization of postural sway on the cortical magnification factor of restricted visual fields. Exp Brain Res 1994;99:501-506.

Tinetti ME, Speechley M, Ginter SF. Risk factors for falls among elderly persons living in the community. N Engl J Med 1988;319:1701-1707.

Wade MG, Jones G. The role of vision and spatial orientation in the maintenance of posture. Phys Ther 1997;77:619-628.

West CG, Gildengorin G, Haegerstrom-Portnoy G, Schneck ME, Lott L, Brabyn JA. Is vision function related to physical functional ability in older adults? J Am Geriatr Soc 2002;50:136-145. 